\title{
CAVITY SEGMENTATION AND STROKE VOLUME MEASUREMENT METHODS FROM ECHOCARDIOGRAPHIC IMAGES: REVIEW
}

\author{
${ }^{1}$ Anas A. Abboud, ${ }^{1}$ Rahmita Wirza Rahmat, ${ }^{2}$ Suhaini Bin Kadiman, \\ ${ }^{3}$ Mohd Zamrin Bin Dimon, ${ }^{1}$ Lili Nurliyana, ${ }^{4}$ M. Iqbal Saripan and ${ }^{1}$ Hasan H. Khaleel \\ ${ }^{1}$ Department of Computer Science and Information Technology, UPM, 43400, Serdang, Malaysia \\ ${ }^{2}$ National Heart Institute (IJN), No 145 Jalan Tun Razak, 50400 Kuala Lumpur, Malaysia \\ ${ }^{3}$ Faculty of Medicine, Universiti Teknologi MARA(UiTM), 47000, Sungai Buloh, Malaysia \\ ${ }^{4}$ Department of Computer and Communication Systems Engineering, \\ Faculty of Engineering, UPM, 43400, Serdang, Malaysia
}

Received 2014-05-06; Revised 2014-06-15; Accepted 2014-11-10

\begin{abstract}
The complexity and criticality of heart disease diagnosis and treatment have frequently attracted the attention of researchers to develop approaches that can handle accurate clinical assessments. One of these complexities is measuring the Right Ventricle stroke volume. Therefore, different cardiac imaging techniques have been developed to help specialists in their regular clinical work and in the operating theatre to assist in the diagnosis, monitoring of the treatment and to ensure the success of a cardiac intervention as far as possible. Echocardiography is one of the cardiac imaging techniques and is considered as the most effective imaging technique as it is minimally invasive, quick and the cheapest method. Many studies have been conducted on Right Ventricle assessment from echocariographic images. This review is aimed at presenting the previous technical elements and algorithms used to measure the ventricle stroke volume from echocardiographic images. The review focuses on the five technical approaches that have been used, which are pre-processing, End-Distally (ED) and End-Systolic (ES) frame detection, cavity segmentation, the stoke volume measurement and 3D reconstruction. Finally, a summary will conclude this study.
\end{abstract}

Keywords: Echocardiography Image, Stroke Volume, Right Ventricle (RV), Left Ventricle (LV), EndDiastolic (ED) and End-Systolic (ES), Three Dimensional Echocardiography (3DE), Preprocessing, Segmentation, 3D illustration, Cloud Point Orientation

\section{INTRODUCTION}

This study presents the review of the approachesutilised in steps of an algorithm presented by (Argones et al., 2008; Rahmat et al., 2013), as a part of research work for cavity segmentation from four Dimension Echocardiographic images (4DE) to measure the Right Ventricle (RV) stroke volume as a part of $\mathrm{PhD}$ thesis in University Putra Malaysia. The paper starts with a block diagramto show the sequence of approaching in the literature review, as illustrated in Fig. 1.

\section{PRE-PROCESSING}

Although the development of the medical imaging techniques and its equipment's and the improvement of the imaging resolution. Pre-processing is still the core the primary step in the medical image processing Corresponding Author: Anas A. Abboud, Department of Computer Science and Information Technology, UPM, 43400, Serdang, Malaysia 
algorithms. This section of the literature is focused on the pre-processing methods performed onechocardiographic images. These methods are performed using simple step (s) or hybrid methods for image enhancement, data size reduction, or processing time reduction.

Echocardiography is a test that uses ultrasound to generate moving pictures of the heart, Bonow et al. (2012). Since ultrasound images can be affected by many factors such as speckle noise, motion artifacts. Dawood et al. (2011) pre-processing is required to obtain high performance in scheme extraction, information retrieval, storage cost reduction, processing time reduction.

Different image enhancement techniques are used to improve the quality of echocardiography images. These include filters, which are used in many studies for image enhancement in the pre-processing stage. High boost filtering was used to enhance high frequency components without affecting the lower frequency components, followed by morphological opening and closing, to reduce the speckle noise. Then, the negative Laplacian filter was used based on kernel convolution for regional filtering to eliminate small contours for the extraction of the intracardial cavity of LV in short axis view images. Sigit et al. (2009a) used a median filter, morphological opening and contrast enhancement to segment the LV contour, then edge detection and image combining for explicit detection. In another study, (Hussein et al., 2009; Lacerda et al., 2008) used a sequence of steps, namely firstly using high-boost filtering, then thresholding and then a Laplacian of Gaussian (LoG) filter for image enhancement in a study to segment the LV cavity. Histogram equalization and an adaptive median filter were used for image enhancement by Deka and Ghosh (2006) followed by morphological dilation and erosion with a group of directional structured structuring elements.

In another study for the segmentation of LV in echo images was done by Santos et al. (2007). Their preprocessing method reduced the data size and processing time through cropping image regions into fixed sizes and then dividing the image into rectangular blocks. The Otsu threshold was then computed for each block and background subtraction was performed.

Frame-based pre-processing is another type of preprocessing approach that can assist in verification performance Argones et al., (2008). Revell et al. (2003) also stated its suitability for feature extraction, specifically to extract the contour of the tendon by creating a composite frame from 20 sequential images. Then, the composite and single frames were subtracted before binarizing the image using empirically determined thresholds to reduce data size. Kondo et al. (1995) used frame-by-frame subtraction to indicate the motion in their assessment of the delayed onset of the LV, where 60 frames/sec echocardiographic images were used. Amorim et al. (2009) used fused three frames in equivalent positions of three cardiac cycles as the first step of pre-processing to enhance LV wall construction and for noise reduction. Then, smoothing and thresholding were applied to improve the segmentation performance.

From this literature we can conclude that the preprocessing can be performed in different methods based on the objective, such as image enhancement which may be used to reduce the noise effects and video frame processing to reduce computation time, feature detection, or it can be used for motion tracking.

\section{RV SEGMENTATION}

Object segmentation is a very important stage in medical image analysis because it affects the assessment accuracy. Boundary detection approaches are among the most frequently used methods for segmenting the cavity in echocardiographic images.

To the best of our knowledge, automatic RV cavity segmentation has not being reported in the literature. Existing approaches required manual initialization of boundary points. This is followed by smooth curve fitting through those points to generate the boundary. Most of the literature deals with cavity segmentation for LVs.

Boundary detection is one of the most frequent methods used to determine the organ feature (Hussein et al., 2009). However, proper detection of boundaries in echocardiography images is difficult due to speckle noise, poor image quality and artifacts caused by intra-cavity structures. Therefore, pre-processing is an important stage in feature extraction for echocardiography images. The pre-processing stage can influence the accuracy of ROI segmentation as well as the time cost of boundary extraction Santos et al. (2007).

The work by Friedland and Adam (1989) used priorknowledge of the geometry of organs to determine the LV cavity, which was considered a fast boundary detection technique. Firstly, low-pass filtering was performed for decimation to reduce the data size and manual rough estimation of the ROI position. A global optimization approach was taken by computing an energy function to determine the suitable edge region. Then, used simulated annealing used to extract the LV boundary.

The initial determination of the ROI position and fuzzy center point estimation of LV at the long and 
short-axis views of a sequence of images from one cardiac cycle was proposed by Setarehdan and Soraghan, (1996) in the first step for boundary extraction of the LV. In the work, the initial boundary points were defined based on the global maximum point of wavelet transformation to defined the candidate points of the cavity boundary, Upon defining the candidate points Cubic B-Spline was employed to generate the smooth LV boundary through these points. The center point and rough locations of endocardial and epicardial boundaries were determined based on the elliptical shape of the LV. The endocardial and epicardial boundary were extracted in sequence of short axis LV images by wavelet-based edge detection approach. Then, the boundary was closed using a local smoothing filter to generate a close smooth boundary Laine and Zong (1996). However, this method failed to extract the boundary from poor quality images. Mishra et al. (2003) used morphological opening and closing with low-pass filtering to optimize the initialization of the genetic algorithm to determine the LV boundary. Santos et al. (2007) presented a fast boundary extraction of LV cavity from long axis view image. Linear stretching of histogram was used for image enhancement. Then, the image was divided into blocks before converting each block into binary images using local Otsu thresholding. Finally, a cubic spline interpolation was used to generate smooth boundaries.

An automated coupled deformable model was used for segmenting the myocardial borders of the LV from RT3D echocardiographic images. This approach was originally proposed by Zhu et al. (2009). It incorporates the incompressibility property of the myocardial and therefore is able to handle the fuzzy epicardial borders. This model maximizes the regional homogeneities of a cardiac image, while maintaining the myocardial volume during a complete cycle. By simultaneously evolving both endocardial and epicardial boundary surfaces, an automatic segmentation of the full myocardium is achieved from RT3D echocardiographic images.

Qin et al. (2013) presented automatic segmentation for RV from four chamber view in $2 \mathrm{DE}$ images, the RV location is detected automatically using training system. The first step of the algorithm was analyzing the statistical information of the images using Sparse Matrix Transform (SMT) to detect the cardiac motion. Secondly, built training model to determine the location of the RV, based on cardiologists' determination of the epicardial and endocardial of the RV cavity on 450 images. Third, the training model was then adjusted as an adapted initialization for the segmentation of each image in the series of one cardiac cycle. Finally, a localized region based level set algorithm was applied based on the adapted initializations, for segmenting the epicardial and endocardial boundaries of the right ventricle, from the whole series of one cardiac cycle images.

Watershed transformation is one of the segmentation methods used to segment the LV cavity. Deka and Ghosh (2006) presented a method to segment the LV cavity in ultrasound image, their approach started with histogram equalization, an adaptive median filter used in preprocessing stage and multiscale morphological gradient method, for image enhancement to achieve better segmentation. The next step was, performing the watershed segmentation method to segment the LV cavity. Finally, they merged the neighbouring regions according to the similarity in region size. The oversegmentation problem was the main limitation of this technique. Therefore, $\mathrm{Ng}$ et al. (2008) aimed to reduce the over-segmentation based on texture and size criteria by computing the angular scan moment, contrast, entropy and inverse difference moment to generate the regional texture map. They computed each segmented region's area to determine the threshold value of each area. Finally, a merging process was applied according to the texture similarity and threshold of region size.

Lacerda et al. (2008) used watershed segmentation to classify the 2D echocardiographic image of the LV to small regions and then these small regions were merged based on empirically identified thresholds of region area of 150 pixels. Next, radial search was used to select the candidate points of the ventricle boundary, short distance interpolation followed by morphological closing to generate the smooth contours.

Sigit et al. (2009b) introduced a boundary segmentation method for the short-axis view of the LV. They began with a high boost filter for image enhancement to reduce the higher frequency components retaining the lower frequency components. This was followed by applying morphological and thresholding operations to eliminate noise and convert the image into binary format. The third step applied the negative Laplacian filter for edge detection. The fourth step was region filtering to eliminate small regions. The last step involved detecting and reconstructing the imprecise border using triangle equation.

Two stages of operation were performed by Junier et al. (2008) to segment the boundary 2D echocardiographic image of the long axis view of LV. In the first stage, images fusion was applied to a sequence of images, followed by smoothing and Otsu thresholding were performed to identify the ROI. Then, the second stage, 
consisted of watershed segmentation to determine the ROI, followed by a multiscale gradient operator to identify the edge of the eroded, the inner and outer boundaries were then determined after filling the regions. This was followed by calculating the Euclidean distance of each region. Finally, watershed segmentation was applied for the image resulted from the Euclidean distance transformation to extract the LV boundary.

Hao et al. (2000) used region growing segmentation for myocardium assessment. Images were classified into classes of gray levels based manually determined points on the myocardium. The myocardium was then segmented into regions using the information characteristics (which represented by characteristic vectors of classes and characteristic vectors of pixels) and geographical similarity of pixels. Mancas et al. (2005) proposed a method to segment the ultrasound images by manual determination of the initial seed point. Subsequently region growing was performed by thresholding the images using a gray space map. Another approach for seed point detection of breast tumour segmentations was presented by Shan et al. (2008) based on a predetermination of the ROI. In this approach, the center point of the ROI was selected as the seed point. According to the literature reviewed in this field, it can be pointed out that, the seed point detection process in most of the method depends on the pre-determination of the ROI, enhancement of the images and a feature description of the segmented ROI (lesion).

As manually selecting seed points can be cumbersome, some studies have proposed automatic seed point detection, such as (Koo et al., 2009). They assumed the pixel value of a tumour image, after undergoing image enhancement, is close to zero.K-means clustering was used for automatic detection of the seed point, thus the grayscale value of the pixels (which assumed to be closed to zero) for the tumour region and the coordinate of the image pixels were considered for the K-mean.

\section{END-DIASTOLIC AND END- SYSTOLIC DETECTION}

Accurate detection of the End-Distally (ED) and EndSystolic (ES) stages of a cardiac cycle is a significant factor that can affect the accuracy of abnormality assessment of a ventricle (Ostenfeld et al., 2012; Darvishi et al., 2012). This process is a routine step of the ventricle assessment procedures, where often, in clinical reports, many parameters can be measured in these two stages to help in diagnosing and decision making Barcaro et al. (2008). However, the process of detecting the ED and ES still a challenge for diagnosis because it was mostly done manually by tracking the cine loop during the clinical procedure, as in Niemann et al. (2007) or by optical tracking of the change of ventricle dimensions through watching the individual image faces of the ventricle during the cardiac cycle, as in (Aune et al., 2009; Crean et al., 2011).

Tracking the mitral valve motion is another method for detecting the ED and ES frame, where the ES stage is defined as the last frame before the opening of the mitral valve while the ED stage is the last frame before the closing for the mitral valve, as reported by Nosir et al. (1996). In the meanwhile, tracking the mitral valve together with the ventricle volume was presented by Barcaro et al. (2008). ECG signal was used by Zhu et al. (2009) to determine the ED and ES frame of the LV in order to generate a dynamic model of the (Gopal et al., 2007; Chua et al., 2009) also used ECG to determine the ED frame through the R wave of the ECG and the ES frame was determined as the smallest cavity area. The biggest and smallest area of the RV cavity were visually tracked in three views; short axis, four chamber and coronal by Ostenfeld et al. (2012) to determine the ED and ES cardiac stages.

Gifani et al. (2010) presented an automatic detection method for ED and ES of LV from two and four chamber views using unsupervised learning algorithm Locally Linear Embedding (LLE) for three cardiac consecutive cycles. Shalbaf et al. (2011) performedimage registration for echocardiographic images of 6 volunteers, distance computation and finally the classical Multi-Dimensional Scaling (MDS) used to construct low dimensional representation of 2D LV images to generate Iso-map. Then, they computed manifold model of seven phases of cardiac cycles to determine ED and ES stages automatically.

Tsui et al. (2013) determined ED and ES stages by tracking the mitral valve motion, based on Nosir et al. (1996)'s definition for the ED and ES stages. This method used 3D echo image of LV, by binaries and enhancing images in the pre-processing stage of their algorithm. Then, a dilation operation for different resolution scale was performed to avoid the blood flow effect. Finally, the number of connecting regions for 8-neighbore connectivity was computed. This method gives accurate results only with good quality echo image of mitral valve.

All of previous methods in this section are time consuming; therefore, an accurate automatic detection for the ED and ES stages is highly required to assist specialists and interns in their clinical work. 


\section{RV VOLUME MEASUREMENT METHODS}

Although, the first historical description of the RV arrhythmic disease was in 1736 by G.M. Lancisi, when he reported the disease in a family recurrence in four generations Basso et al. (2009). The first electrocardiographic description of the RV disease was in 1961, two cases reported by Sergio et al. (1961) for auricularisation which is "the right ventricular pressure curve" and the main pulmonary artery pressure through the characteristic epsilon wave. Since then, the disease has been noted as the main cause of sudden death in youth and particularly in athletes Fontaine and Frank (1984).

Later, cardiac imaging techniques such as MRI, CCT and echocardiography are developed to visualize moving heart structure and provide a wealth of information and measurements. Echocardiography is one of these techniques, which became one of routine clinical tool for diagnosing, assessing, pre-operation planning and treating. There are many studies presented to assess the $\mathrm{RV}$ volume and function.

Levine et al. (1984) pioneered the assessment when they measured the RV volume of latex model using the apical four-chamber view and subcostal RV outflow tract view. The endocardial boundary determined by Microsonic computer and the disconnected boundary joined by straight lines. The measurement was done by measuring the area of one viewing plane $(A 1)$ and the length of the longest axis ( $L 2)$, then the volume was computed by the mathematical relation Equation (1):

$V=c \times A_{1} \times L_{2}$

where, $c$ is a constant.

Meanwhile, (Starling et al., 1982; Helbing et al., 1995) used Disk Summation Methods (DSM) or Simpson rule to measure the RV volume, for a predominantly fourchamber view in 2D images, the Ventricle Outflow Tract (RVOT) was excluded from the volume. Therefore, this method gave an underestimated volume.

$\mathrm{RV}$ apical four chamber view is considered by Foale et al. (1986) as the best view that could be used to defined the apex and the tricuspid valve of the RV, in their study to assess the right ventricular function from the area of the RV at the ED and ES frames, using off-line computer system produced by Microsonics Company. The ED and ES frames were identified by the closure and opening of the tricuspid valve.

Area-length methods is another method used with 2D echocardiography images. This method requires geometrical approximation of RV structure based on ellipsoidal or modified pyramidal model after manual determination for the intracardial boundary Leng et al. (1994). As it was reported in many previous researches, the results of measuring the RV volume in $2 \mathrm{D}$ images were not accurate, while this method is based on geometrical assumptions; therefore, it is very sensitive to the imaging plane and angle.Recently, the Three-Dimensional Echocardiography (3DE) technology, utilized fully sampled matrix array transducers with live 3D mode. This technology displays a pyramid of 3D images of the cardiac scheme and allows a complete visualization of the entire myocardium (Vegas and Meineri, 2010), those 3D image scans be obtained from a single acquisition using one wide imaging mode for one cardiac cycle or from several consecutive cardiac cycles using four or seven narrow neighbored angles. The improvement in $3 \mathrm{D}$ imaging comes from the availability and accessibility to RT3DE. However, RV size and function quantification remains clinically difficult despite of the advanced cardiac imaging.

There are many researchers who published their works on measuring RV volume using 3D techniques. Among these are Aune et al. (2009), who used QLAB and applied Left Ventricle (LV) measuring method, by determining four landmarks at the tricuspid valve annulus and one at the apex on the apical view of the RV, then the QLAB quantification software determined the boundary based on the selected landmarks, to build the 3D model. Aune's method excluded the pulmonary valve resulting in an undesirable ellipsoid 3D shape output, which does not cover the trabecular nature of RV. Therefore, the volume was inaccurate. Earlier, Marwick et al. (2007) tracked the RV endocardial contours by semi-automatic border detection after determining the tricuspid annulus and apex for 12 long axis slices of the RV, a preconfigured ellipse was fitted into the endocardial boundary (which was used for left ventricle model) then applied on the ventricle wall with modification to fit the boundary with the endocardial border. Hoch et al. (2007) calculated the RV volume of RV latex model for the heart of lambs. The data was acquired using single wide-angle imaging. The volume measured by DSM using Tom Tec software http://www.tomtec.de/on a short-axis cutting plane. The gain was optimized to clearly visualize the endocardial rim. The disk thickness was $5 \mathrm{~mm}$ and then the process was repeated for few times. At each time, they changed one of the three elements setting (disk orientation, gain, disk thickness). Then, the result of each setting was compared with the previous. This study concluded that $\mathrm{RV}$ volume measurement could be affected by the slicing direction, the slice thickness (number of disks) and the 
gain. We should mention that this study did not include the outflow tract in the volume measurement.

Gopal et al. (2007; Horton et al., 2009; Chua et al., 2009), however, used Tom Tec system to measure the RV volume. 3D full-volume reconstructed surface from four wedges and RV boundary were determined manually to overcome the drop out of the inflow and outflow tract. Then 3D surface model was built using the method proposed by Weiss et al. (1982) for the Beulet 3D reconstructed surface. This method was used to reconstruct and measure the LV volume as well. Meanwhile, DSM method of $10 \mathrm{~mm}$ disk thickness with different width and length for each disk used as a second method by Gopal et al. (2007) in their study to compare the results with CMR technique. The boundary was determined manually at each plane. Wide angled full volume imaging from modified apical four-chamber view was used for imaging the RV cavity and DSM used to measure the stroke volume. This study has reported many limitations caused by the difference between the real anatomy and reconstructed surface. Such limitations are the imaging angle and the view caused a disappearance to the inflow and outflow tract and failed to determine the RV boundary accurately.

Sugeng et al. (2010) acquired RV from apical window using four consecutive gated cardiac cycle to reconstruct full-volume data. Manual initialization of contours was performed at one mid-ventricular short axis plane, apical four-chamber plan and coronal plan to create the 3D model. The ED and ES frames were determined manually. The volume was measured through the reconstructed surface model. This study concluded that the DSM obtained from 2D fundamental was inaccurate measurement method for RV volume. Earlier, Niemann et al. (2007) measured the RV volume from a full volume dataset. The RV cavity endocardial boundary determined through three views, the sagittal view plane to outline the tricuspid valve, four-chamber view used to outline the apex and a coronal view to outline the RV Outflow Tract (RVOT). The volume has been computed at the ED and ES frames by the summation of each slice area from a full volume dataset, including semi-automatic border detection with manual correction to build the 3D model. ED and ES frames were determined manually depending on the cineloop images of the cardiac cycle; this measurement included 12 to 24 slices from the interventricular annulus to the ventricular outflow tract and excluded the interventricular septum and the apical component of the $\mathrm{RV}$ moderator band from the calculation.

In a comparison study of the RV volume measurement of using 3DE and MRI imaging, Crean et al. (2011) acquired RV from a modified four-chamber imaging view to maximize the visualization of $\mathrm{RV}$. The volume reconstruction was performed from 2D multiplanar view for semi-automatic intracardial boundary detection with manual correction. The ED and ES were selected manually by observing the individual image phases. Finally, the volume was measured based on the reconstructed $3 \mathrm{D}$ surface.

From the previously published literature concerning RV volume measurements, we noticed that most involved intensive manual interaction for RV contour detection. Likewise, those previous measurement methods adopted multi-view images in their approaches. This issue requires optimizing rotation angulation and manipulation to get the optimum view and enable boundary-tracing process. The $2 \mathrm{D}$ method fails to measure the RV volume because it is based on geometrical assumptions. However, many iterations were required to model a full structure of the RV. In addition, the Disk Summation (DS) approximation, which is an extension of a fundamentally $2 \mathrm{D}$ technique, fails to take full advantage of the RT3DE images that contain the entire ventricle, because of the failure of identifying the outflow tract accurately and include it in computing the volume of the RV cavity. Single wide-angle full-volume imaging of RV can cause disappearance of the inflow and outflow tract especially with dilated RVs.

\section{3D ILLUSTRATION OF RV ANATOMY}

$3 \mathrm{D}$ reconstruction is a vital approach in the medical field because it can provide more information leading to better analyses of human organs. The reconstruction models can be used to assist diagnostics and robotic surgery. However, the medical field is always open for challenges and there is always a material for research. There are different surface reconstruction methods presented for different data set type. In this section, the most frequent surface reconstruction methods used to reconstruct the $3 \mathrm{D}$ model of the heart ventricles from echocardiographic images are reviewed.

To the best of our knowledge, segmentation techniques used in medical imaging treat the extraction of the organ boundary as the most crucial step to assess and diagnose the organ status. Therefore, contour-based surface reconstruction techniques are widely used for medical images and give accurate results in organ surface reconstruction Qiang et al. (2007). In this technique, the cloud point data set $\mathbb{R}^{3}$ generated from the coordinates of the contour points of the extracted region from the slices are used in surface reconstruction. In the most frequent cases, either the input data set $\mathbb{R}^{3}$ requires pre-processing to generate accurate surface, or the reconstructed surface requires enhancement or post- 
processing to satisfy the accuracy and fairness of the final shape. To reconstruct smooth surface from a set of parallel binary contours, (Braude et al., 2007; Marker et al., 2006) used the Multi-level Partition of Unity (MPU) approach to reconstruct an implicit surface for contour of large point set successfully. This approach focused on the local features and details even though it may show sampling/subdivision artifacts. Another approach was introduced by Qiang et al. (2007), where they developed the common known method Radial Based Function (RBF) interpolation to Localby adding forward and backward slices to keep the consistency of the surface. This technique was used for reconstructing surfaces from large datasets. Triangulation is also used to create meshes directly from the contours. This method is useful for convex contours only. Based on surface evaluation, Sahillioğlu and Yemez (2006) used surface deformation to produce a smooth and accurate surface. This technique overcomes the fold-overs and non-manifold triangulations problems. Miller et al. (1991), presented a deformable method to reconstruct close 3D surface of polygon, using a balloon model as a 'seed' model and deformed this balloon until it complies with the object surface (3D CT data used in this study). Cohen et al. (1992); Mcinerney and Terzopoulos, 1995) implemented an elastically deformable cylinder and sphere, respectively used finite element and physical-based techniques for anatomical based segmentation, reconstruction and tracking structures from multidimensional CT and MRI images. The surface consists of the triangular finite element model, which included position and first and second parametric partial derivatives of the surface. The model deformed to fit the data in an elegant and intuitive manner based on Lagrangian equations of motion, that adjust the model's deformational degrees of freedom to fit the LV data. This method worked successfully with multi-dimensional image slices that do not suffer from the banding artifacts.

\section{DATA ORIENTATION}

Point clouds by themselves are not useful to process the data and make measurements and other calculations. In addition, some data redundancy problems may exist in scanned data. This makes orienting unorganized cloud points crucial in surface reconstruction to get the correct connectivity for the sample points and identifying the inside/outside points. However, generating correct oriented vector in regions with very sparse points is a challenge.

Liu and Wang (2010) presented their Orienting approach (ORT) in the pre-processing step for surface reconstruction. This approach were performed through three main steps, in the first step, a sphere splitting step based on eigenvalue analysis were added, to a modified scheme of generating adaptive spherical cover for unorganized points. Generated the triangular mesh surface and cleaned its topology, to find the closest triangle to every input points and then specify their orientations using a local search based algorithm, in the second step. Lastly, an orientation-aware principle component analysis step gives correct and consistently oriented normal vectors to the unorganized input points.

Adaptive Spherical Cover (ASC) was used to compute the oriented vector of the points nearest to the triangular mesh surface introduced earlier by Ohtake et al. (2005). Then, the oriented vectors are assigned to the unorganized points using Principle of Component Analysis (PCA). This technique doesn't modify the point cloud. Earlier, Amenta and Bern (1999) developed an algorithm for surface reconstruction of scattered and unorganized $3 \mathrm{D}$ data. The algorithm, which was based on a Voronoi diagram, guaranteed good results when a good sample of data was given. A good sample means capturing of intuitive notions to enable the areas with few samples.

\section{CONCLUSION AND DISCUSSION}

From the previous published literature concerning RV volume measurements, we noticed that measuring the RV volume still required intensive manual interaction for RV contour detection. Likewise, those previous measurement methods adopted multi-view images in their approaches. This issue requires optimizing rotation angulation and manipulation to get the optimum view and enable boundary-tracing process.

Disk Summation (DS) approximation, which is an extension of a fundamentally $2 \mathrm{D}$ technique, was one of the methods used to measure the RV volume. This method fails to take full advantage of the RT3DE images that contain the entire ventricle, because of the failure to identify the outflow tract accurately and include it in computing the volume of the RV cavity. Model-based measurement methods were also used by some researchers to measure the cavity volume from the $3 \mathrm{D}$ model that reconstructed from the cavity boundary. These methods however ignore the actual intracavity features and that leads to inaccurate results.

For segmentation, as shown in Fig. 1, different approaches were utilized. We can summarize it as boundary detection method with prior knowledge for ventricle feature regional segmentation method which used texture and watershed segmentation these methods extracted endocardial boundary, to reconstruct the 3D model. 


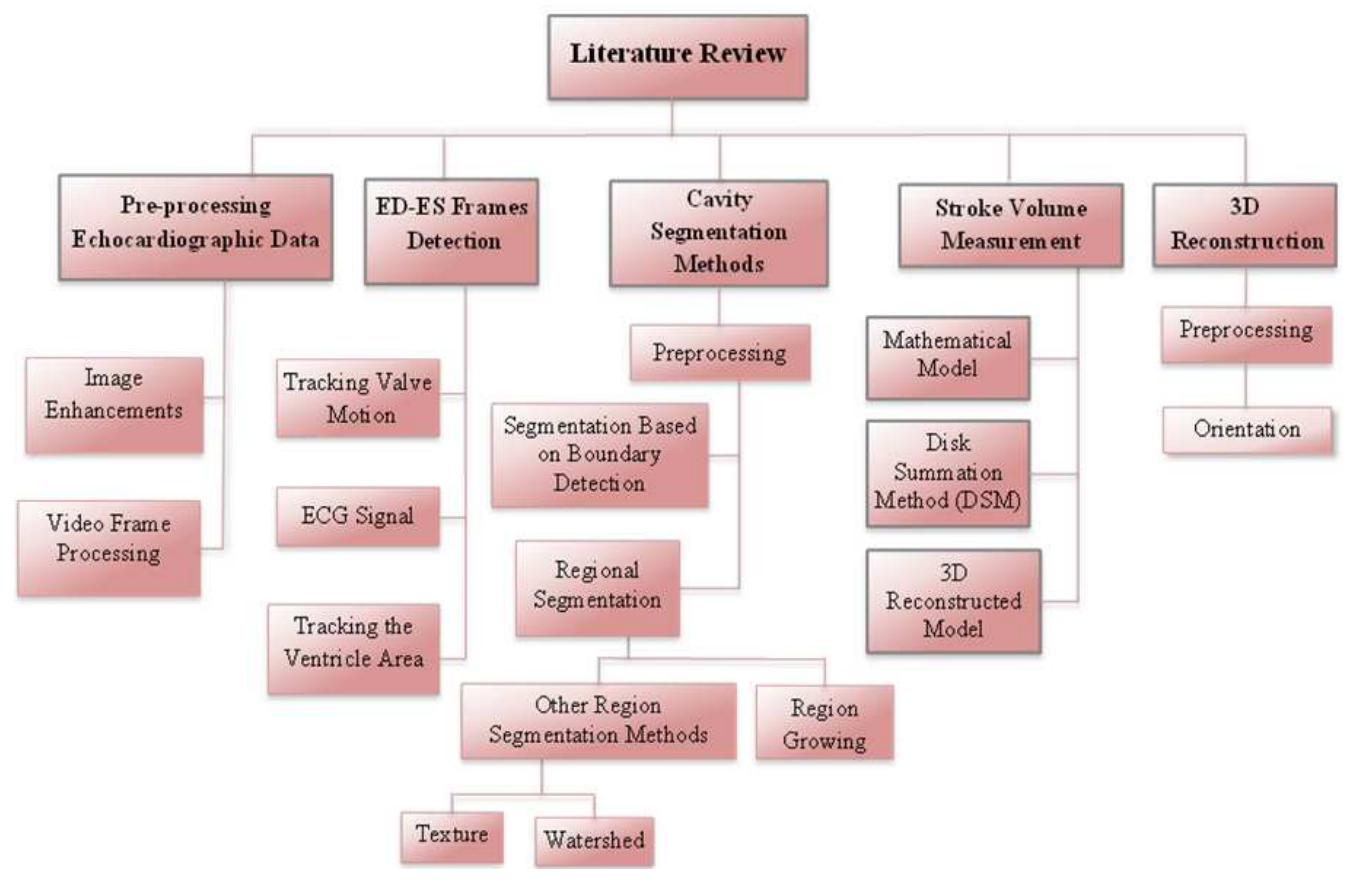

Fig. 1. Block diagram showing the reviewed approaches in this study

Meanwhile, the ED and ES frame detection is still done manually and depends on the user experience, which may effect on the accuracy of measurement results of the cavity stroke volume. Finally, the most frequent $3 \mathrm{D}$ reconstruction methods used with echocardiography images are reviewed.

It is obvious that there is a lack of concentration on the multifarious structure of the RV cavity (apex, moderator band, trabecular and inflow-outflow regions), in additional to the difficulty of identifying the endocardial boundary accurately in the End-Systolic stage of the RV due to more densely packed trabeculations and papillary muscles. Therefore, new algorithms are needed to assess the abnormality of the right ventricle. This process can be done by accurate segmentation of the cavity, determination of the EndDiastolic and End-Systolic stages of the cardiac cycle, measuring the stroke volume and reconstructing the three dimension model of the segmented region of the cavity for initial assessment of the abnormality.

Thus, we propose a method for semi-automatic segmentation of the right ventricle to measure the stroke volume from four Dimensions (4D) echocardiography, based on a novel analysing for the complex geometrical structure and function of the right ventricle, (Rahmat et al., 2013; Anas et al., 2014).
The right ventricle structure is simplified by slicing the right ventricle in 4D echocardiography images. Region growing technique is deployed to segment the cavity in each slice. This technique works automatically by detecting a seed point inside the Region of Interest (ROI), independently utilizing pre-knowledge of the region feature. Then start the iterative region growing segmentation process. Automatic detection for the EndDiastolic and End-Systolic stages of the cardiac cycle is introduced, by tracking the changes area of the segmented region of the cavity along one cardiac cycle. Disk summation principle is used to compute the volume of the segmented region in each slice. The resolution of the xMATREX array TEE transducer (X7-2t) is also estimated to measure the volume in millilitre unit. Then compute the cavity stroke volume by finding difference between the volume of the cavity at the ED and ES stages. The contours of the segmented region are extracted to generate the cloud of points $(\mathbb{R} 3)$. Finally, generate three dimensions modelling for the segmented cavityby developing the normal feature approximation method for the cloud point, in order to accurately delineate the required object.

The proposed method requires minimal userinitialization to determine the ROI and executions, which requires only few seconds for one time along the processing time. Comparisons of the segmentation, end- 
diastolic and end-systolic stages, stroke volume and the reconstructed 3D model; are provided with currently available software for left ventricle volume, function assessment and 3D modelling to validate the merit of the proposed work.The results of each step of process are satisfied high acceptance from the cardiologist experts in the qualitative validation and a good accuracy in quantitative validation regarding to techniques.

\section{ACKNOWLEDGMENT}

The researchers wish to thank the Heart and Lung Centre in PPUKM for providing us research time on the $\mathrm{X}$-Ray imaging system, angiograms and echocardiograph images. We also thank all those lovely girls, Yan Yin Fong, Swee Lee Ping and Wei Bey Fenh for helping us implementing the system. This study was granted under Prototype Research Grant of Malaysia Minister of Higher Education with the title of "Visualization and Diagnostic of Coronary Arterial Stenosis through Voice Activation". This study was supported in part by Universiti Putra Malaysia, Faculty of Computer Science and Information Technology, Department of Multimedia under Fitting nonUniform B-Spline Curve and Surface to Transesophageal Echocardiography Images to Determine and Abnormality of Cardiac Structure, FRGS, Project code:03-04-10868FR, 24 months, April 2010-2012, RM44K grant.

\section{REFERENCES}

Amenta, N. and M. Bern, 1999. Surface reconstruction by Voronoi filtering. Discrete Computat. Geomet., 22: 481-504. DOI: 1007/PL00009475

Amorim, J.C., M.D.C. Dos Reis, J.L.A. De Carvalho, A.F. Da Rocha and J.F. Camapum, 2009. Improved segmentation of echocardiographic images using fusion of images from different cardiac cycles. Proceedings of the IEEE Annual International Conference of the Engineering in Medicine and Biology Society, Sept. 3-6, IEEE Xplore Press, Minneapolis, MN, pp: 511-14. DOI: 10.1109/IEMBS.2009.5333101

Anas, A., W.R. Rahmita, B.K.M. Suhaini, M. Iqbal and H. Hasan, 2014. Automatic detection of the enddiastolic and end-systolic from 4d echocardiographic images. J. Comput. Sci., 1: 230240. DOI: $10.3844 /$ jessp.2015.230.240

Argones, R.E., C.J.L. Alba and M.C. García, 2008. Quality-based score normalization and frame selection for video-based person authentication. Biomet. Identity Manag., 5372: 1-9. DOI: 10.1007/978-3-540-89991-4_1
Aune, E., M. Baekkevar, O. Rodevand and J.E. Otterstad, 2009. The limited usefulness of real-time 3-dimensional echocardiography in obtaining normal reference ranges for right ventricular volumes. Cardiovascular Ultrasound. DOI: 10.1186/1476-7120-7-35

Barcaro, U., D. Moroni and O. Salvetti, 2008. Automatic computation of left ventricle ejection fraction from dynamic ultrasound images. Patt. Recognit. Image Anal., 18: 351-358. DOI: 10.1134/S1054661808020247

Basso, C., D. Corrado, F.I. Marcus, A. Nava and G. Thiene, 2009. Arrhythmogenic right ventricular cardiomyopathy. 373: 1289-1300. DOI: 10.1016/S0140-6736(09)60256-7

Bonow, R.O., T.G. Ganiats, C.T. Beam, K. Blake and D.E. Casey et al., 2012. ACCF/AHA/AMA-PCPI performance measures for adults with heart failure a report of the american college of cardiology foundation/american heart association task force on performance measures and the american medical association-physician consortium for performance improvement. J. Am. Coll. Cardiol., 59: 1812-1832. DOI: 10.1016/j.jacc.2012.03.013

Braude, I., J. Marker, K. Museth, J. Nissanov and D. Breen, 2007. Contour-based surface reconstruction sing MPU implicit models. Graph Models, 69: 139157. DOI: 10.1016/j.gmod.2006.09.007

Chua, S., A.R. Levine, C. Yosefy, D.M. Handschumacher and J. Chu et al., 2009. Assessment of right ventricular function by real-time three-dimensional echocardiography improves accuracy and decreases interobserver variability compared with conventional two-dimensional views. Eur. J. Echocardiograp., 10: 619-624. DOI: 10.1093/ejechocard/jep013

Cohen, I., L.D. Cohen and N. Ayache, 1992. Usingdeformable surfaces to segment 3-D images and infer differential structures. Proceedings of the 2nd European Conference on Computer Vision Santa Margherita Ligure, May 19-22, Italy, pp: 648652. DOI: 10.1007/3-540-55426-2_69

Crean, A.M., N. Maredia, G. Ballard, R. Menezes and G. Wharton et al., 2011. 3D Echo systematically underestimates right ventricular volumes compared to cardiovascular magnetic resonance in adult congenital heart disease patients with moderate or severe RV dilatation. J. Cardiovascular Magnet. Resonan., 13: 7878. DOI: $10.1186 / 1532-429 X-13-78$ 
Darvishi, S., H. Behnam, M. Pouladian and N. Samiei, 2012. Measuring left ventricular volumes in twodimensional echocardiography image sequence using level-set method for automatic detection of end-diastole and end-systole frames. Res. Cardiovasc Med., 1: 39-45. DOI: 10.5812/cardiovascmed.6397

Dawood, F.A.A., R.W. Rahmat, M.Z. Dimon, L. Nurliyana and S.B. Kadiman, 2011. Automatic boundary detection of wall motion in two-dimensional echocardiography images. J. Comput. Sci. DOI: 10.3844/jcssp.2011.1261.1266

Deka, B. and D. Ghosh, 2006. Watershed segmentationfor medical ultrasound images. Proceedings of the IEEE International Conference on Systems, Man and Cybernetics, Oct. 8-11, IEEE Xplore Press, Taipei, pp: 3186-3191. DOI: 10.1109/ICSMC.2006.384607

Foale, R., P. Nihoyannopoulos, W. McKenna, A. Kleinebenne and A. Nadazdin et al., 1986. Echocardiographic measurement of the normal adult right ventricle. British Heart J., 56: 33-44. DOI: 10.1136/hrt.56.1.33

Fontaine, G. and R. Frank, 1984. Signification des troubles de conduction intraventriculaires observés dans la dysplasie ventriculaire droite arhythmogène. Arch. Mal. Coeur, 77: 872-879.

Friedland, N. and D. Adam, 1989. Automatic ventricular cavity boundary detection from sequential ultrasound images using simulated annealing. Medical Imag., IEEE Trans., 8: 344-353. DOI: $10.1109 / 42.41487$

Gifani, P., H. Behnam, A. Shalbaf and Z.A. Sani, 2010. Automatic detection of end-diastole and end-systole from echocardiography images using manifold learning. Physiol. Measurem., 31: 1091-1091. DOI: 10.1088/0967-3334/31/9/002

Gopal, A.S., O. CEbere, C.J. Iwuchukwu, K.S. Alan and N. Reichek, 2007. Normal values of right ventricular size and function by real-time 3-dimensional echocardiography: Comparison with cardiac magnetic resonance imaging. J. Am. Soc. Echocardiog., 20: 445-455. DOI: 10.1016/j.echo.2006.10.027

Hao, X., C. Bruce, C. Pislaru and J.F. Greenleaf, 2000. A novel region growing method for segmenting ultrasound images. Paper presented at the Ultrasonics Sym., 2: 1717-1720. DOI: 10.1109/ULTSYM.2000.921653
Helbing, W.A., H.G. Bosch, C. Maliepaard, S.A. Rebergen and R.J. Van der Geest, 1995. Comparison of echocardiographic methods with magnetic resonance imaging for assessment of right ventricular function in children. Am. J. Cardiol., 76: 589-594. DOI: 10.1016/S0002-9149(99)80161-1

Hoch, M., V.V. Nikolay, B. Soriano, K. Gauvreau and R.G. Marx, 2007. Variables influencing the accuracy of right ventricular volume assessment by real-time 3-dimensional echocardiography: An in vitro validation study. J. Am. Soc. Echocardiog., 20: 456-461. DOI: 10.1016/j.echo.2006.10.017

Horton, K.D., R.W. Meece and J.C. Hill, 2009. Assessment of the right ventricle by echocardiography: A primer for cardiac sonographers. J. Am. Soc. Echocardiog., 22: 776792. DOI: 10.1016/j.echo.2009.04.027

Hussein, Z.R., R.W. Rahmat, L. Nurliyana, M.I. Saripan and M.Z. Dimon, 2009. Pre-processing importance for extracting contours from noisy echocardiographics images. Int. J. Comput. Sci. Netw. Sec., 9: 134-137.

Junier, W.M., C.R. Cardoso, G.P. Garlet, G.E. Crippa and A.L. Rosa et al., 2008. Evidence of the presence of $\mathrm{T}$ helper type 17 cells in chronic lesions of human periodontal disease. Oral Microbiol. Immunol., 24: 1-6. DOI: 10.1111/j.1399-302X.2008.00463.x

Kondo, H., T. Masuyama, K. Ishihara, T. Mano and K. Yamamoto, 1995. Digital subtraction high-framerate echocardiography in detecting delayed onset of regional left ventricular relaxation in ischemic heart disease. Circulation, 91: 304-312. DOI: 10.1161/ 01.CIR.91.2.304

Koo, L.J., K. Min-Suk, J. Hee-Won, P. Sang-Chul and W. Gi-Nam, 2009. Seed point detection of multiple cancers based on empirical domain knowledge and k-means in ultrasound breast image. Proceedings of the International Conference, Nov. 7-8, Shanghai, China, pp: $507-$ 516. DOI: 10.1007/978-3-642-05253-8_56

Lacerda, S.G., A.F. Da Rocha, D.F. Vasconcelos, J.L. De Carvalho and I.G. Sene, 2008. Left ventricle segmentation in echocardiography using a radialsearch-based image processing algorithm. Conf. Proc. IEEE Eng. Med. Biol. Soc., DOI: 10.1109/IEMBS.2008.4649130

Laine, A. and X. Zong, 1996. Border identification of echocardiograms via multiscale edge detection and shape modeling. IEEE Conf. Publicat., 3: 287-290. DOI: 10.1109/ICIP.1996.560486 
Leng, J., S. Samuel, H. Mark, G.J. Luis and V.D. Prada, 1994. Myocardial Imaging: Three-Dimensional Echocardiography. In: Vivo Validation for Right Ventricular Volume and Function, Association, A.H., (Ed.), USA, pp: 2342-2350.

Levine, R.A., T.C. Gibson, T. Aretz, L.D. Gillam and D.E. Guyer, 1984. Echocardiographic measurement of right ventricular volume. Circulation, 69: 497505. DOI: 10.1161/01.CIR.69.3.497

Liu, S. and C.C.L. Wang, 2010. Orienting unorganized points for surface reconstruction. Proceedings of the IEEE International Conference on Shape Modeling and Applications, (SMA' 10), Pergamon Press, Inc., pp: 1-11. DOI: 10.1016/j.cag.2010.03.003

Mancas, M., B. Gosselin and B. Macq, 2005. Segmentation using a region-growing thresholding. Proceedings of the Image Processing: Algorithms and Systems, (AS' 05). SPIE, DOI: $10.1117 / 12.587995$

Marker, J., I. Braude, K. Museth and D. Breen, 2006. Contour-based surface reconstruction using implicit curve fitting and distance field filtering and interpolation. Graphics.

Marwick, H.T., C. Jenkins, J. Chan, K. Bricknell and M. Strudwick, 2007. Reproducibility of right ventricular volumes and ejection fraction using real-time threedimensional echocardiography. CHEST, 131: 18441851. DOI: $10.1378 /$ chest.06-2143

McInerney, T. and D. Terzopoulos, 1995. A dynamic finite element surface model for segmentation and tracking in multidimensional medical images with application to cardiac 4D image analysis. Comput. Med. Imag. Graph., 19: 69-83. DOI: 10.1016/08956111(94)00040-9

Miller, J.V., D.E. Breen, W.E. Lorensen, R.M. O'Bara and M.J. Wozny, 1991. Geometrically deformed models: A method for extracting closed geometric models form volume data. SIGGRAPH Comput. Graph., 25: 217-226. DOI: 10.1145/127719.122742

Mishra, A., P.K. Dutta and M.K. Ghosh, 2003. A GA based approach for boundary detection of left ventricle with echocardiographic image sequences. Image Vision Comput., 21: 967-976. DOI: 10.1016/S0262-8856(03)00121-5

Ng, H.P., S. Huang, S.H. Ong, K.W.C. Foong and P.S. Goh, 2008. Medical image segmentation using watershed segmentation with texture-based region merging. Proceedings of the 30th Annual International Conference of the IEEE Engineering in Medicine and Biology Society, Aug. 20-25, IEEE Xplore Press, Vancouver, BC, pp: 4039-4042. DOI: 10.1109/IEMBS.2008.4650096
Niemann, P.S., L. Pinho, T. Balbach, C. Galuschky and M. Blankenhagen, 2007. Anatomically oriented right ventricular volume measurements with dynamic three-dimensional echocardiography validated by 3 -tesla magnetic resonance imaging. J. Am. College Cardiol., 50: 1668-1676. DOI: 10.1016/j.jacc.2007.07.031

Nosir, Y.F., P.M. Fioretti, W.B. Vletter, E. Boersmaand A. Salustri., 1996. Accurate measurement of left ventricular ejection fraction by three-dimensional echocardiography a comparison with radionuclide angiography. Circulation, 94: 460-466. DOI: 10.1161/01.CIR.94.3.460

Ohtake, Y., A. Belyaev and H.P. Seidel, 2005. An integrating approach to meshing scattered point data. Proceedings of the ACM Symposium on Solid and Physical Modeling, Jun. 13-15, New York, pp: 6169. DOI: $10.1145 / 1060244.1060252$

Ostenfeld, E., M. Carlsson, K. Shahgaldi, A. Roijer and J. Holm, 2012. Manual correction of semi-automatic three-dimensional echocardiography is needed for right ventricular assessment in adults; validation with cardiac magnetic resonance. Cardiovasc Ultrasound. DOI: 10.1186/1476-7120-10-1

Qiang, W., Z. Pan, C. Chun and B. Jiajun, 2007. Surface rendering for parallel slices of contours from medical imaging. Comput. Sci. Eng., 9: 32-37. DOI: 10.1109/MCSE.2007.18

Qin, X., Z. Cong and B. Fei, 2013. Automatic segmentation of right ventricular ultrasound images using sparse matrix transform and a level set. Phys. Med. Biol., 58: 7609-24. DOI: 10.1088/00319155/58/21/7609

Rahmat, R.W., A.A. Abboud, S.B. Kadiman, M.Z. Dimon and L. Nurliyana, 2013. Experimentation of measuring three dimensional ultrasound resolution. Proceedings of the World Congress on Engineering and Computer Science, Oct. 23-25, San Francisco, USA.

Revell, J.D., M. Mirmehdi and D. McNally, 2003. Applied review of ultrasound image feature extraction methods. Europe PubMed Central.

Sahillioğlu, Y. and Y. Yemez, 2006. A surface deformation framework for $3 \mathrm{D}$ shape recovery. Proceedings of the International Workshop Multimedia Content Representation, Classification and Security, Sept. 11-13, Istanbul, Turkey, pp: 570577. DOI: 10.1007/11848035_75

Santos, J.B., D. Celorico, J. Varandas and J. Dias, 2007. Automatic segmentation of echocardiographic left ventricularimages by windows adaptive thresholds. Proceedings of the International Congress on Ultrasonics, Apr. 9-13, Vienna, pp: 1-4. DOI: 10.3728/ICUltrasonics.2007.Vienna.1680_santos 
Sergio, D.V., B. Gino and Z. Ennio, 1961. Auricularization" of right ventricular pressure curve. Am. Heart J., 61: 25-33. DOI: 10.1016/00028703(61)90513-0

Setarehdan, S.K. and J.J. Soraghan, 1996. Automatic left ventricular feature extraction and visualisation from echocardiographic images. Proceedings of the Computers in Cardiology, Sep. 8-11, IEEE Xplore Press, Indianapolis, pp: 9-12. DOI: 10.1109/CIC.1996.542460

Shalbaf, A., H. Behnam, P. Gifani and Z. Alizadeh-Sani, 2011. Automatic detection of end systole and end diastole within a sequence of 2-D echocardiographic images using modified Isomap algorithm. Proceedings of the Middle East Conference on Biomedical Engineering, Feb. 21-24, IEEE Xplore Press, Sharjah, pp: 217-220. DOI: 10.1109/MECBME.2011.5752104

Shan, J., H.D. Cheng and Y. Wang, 2008. A novel automatic seed point selection algorithm for breast ultrasound images. Proceedings of the 19th International Conference on Pattern Recognition, Dec. 8-11, IEEE Xplore Press, Tampa, FL, pp: 1-4. DOI: 10.1109/ICPR.2008.4761336

Sigit, R., M.M. Mustafa, A. Hussain, O. Maskon and I.F.M. Noh, 2009a. Automatic border detection of cardiac cavity images using boundary and triangle equation. Proceedings of the IEEE Region 10 Conference, Jan. 23-26, IEEE Xplore Press, Singapore, pp: 1-4. DOI: 10.1109/TENCON.2009.5395808

Sigit, R., M.M. Mustafa, O.M. Aini Hussain and I.F.M. Noh, 2009b. Automatic segmentation of cardiac cavity images using collinear and triangle equation. Technol. Devel. Network., Educ. Automat. DOI: 10.1007/978-90-481-9151-2_61
Starling, M.R., M.H. Crawford, S.G. Sorensen and R.A. O'Rourke, 1982. A new two-dimensional echocardiographic technique for evaluating right ventricular size and performance in patients with obstructive lung disease. Circulat. Am. Heart Associat., 66: 612-620. DOI: 10.1161/01.CIR.66.3.612

Sugeng, L., V. Mor-Avi, L. Weinerl, J. Niel and C. Ebner, 2010. Multimodality comparison of quantitative volumetric analysis of the right ventricle. J. Am. Coll. Cardiol. Img., 3: 10-18. DOI: 10.1016/j.jcmg.2009.09.017

Tsui, G.K., K.Y.K. Wong and A.P. Lee, 2013. Automatic systole-diastole classification of mitral valve complex from RT-3D echocardiography based on multiresolution processing. Med. Imag., SPIE. DOI: 10.1117/12.2006611

Vegas, A. and M. Meineri, 2010. Three-dimensional transesophageal echocardiography is a major advance for intraoperative clinical management of patients undergoing cardiac surgery: A core review. Int. Anesthesia Res. Soc., 110: 1548-1573. DOI: 10.1213/ANE.0b013e3181d41be7

Weiss, J.L., L.W. Eaton, C.H. Kallman and W.L. Maughan, 1982. Accuracy of volume determination ejecting canine left ventricle. Circulation, 67: 889895. DOI: 10.1161/01.CIR.67.4.889

Zhu, Y., X. Papademetris, J.A. Sinusas and J.S. Duncan, 2009. A dynamical shape prior for LV segmentation from RT3D echocardiography. Med. Image Comput. Assist Interv., 5761: 206-213. PMID: 20054422 